\title{
RESIDUATED LATTICES*
}

\author{
BY \\ MORGAN WARD AND R. P. DILWORTH
}

\section{INTRODUCTION}

1. We propose to develop here a systematic theory of lattices $\nmid$ over which an auxiliary operation of multiplication or residuation is defined. We begin by showing that the two operations correspond to one another; under quite general conditions in every lattice over which a multiplication is defined a residuation may be defined and conversely. The residuation and multiplication we introduce have the properties of the like-named operations in the particular instance of polynomial ideal theory.

We next give various necessary conditions and sufficient conditions that such operations may exist in an arbitrary lattice, and apply our results to projective geometries and Boolean algebras.

In the third division of the paper we extend E. Noether's decomposition theorems of the ideal theory of commutative rings to general lattice theory. The introduction of a multiplication is obviously necessary for such a generalization. The surprising result emerges that the decomposition theorems are largely independent of the modular axiom, as we show by specific examples. We take this occasion to correct an error made in the preliminary account of our researches (Ward and Dilworth [1]). Since we wrote this, we have obtained many new results which we give here for the first time.

We plan to describe the main part of our investigations of distributive residuated lattices elsewhere (Ward and Dilworth [1], \$\$5, 6). Here we settle some questions raised by one of us (Ward [1]) as to the significance of certain auxiliary conditions which a residuation may satisfy by showing in all cases that they imply that the lattice is distributive.

2. It was not until this paper was virtually completed that we learned of the investigation of Krull upon this subject (Krull [1]). There is, however, very little duplication between our results and Krull's. Krull was chiefly concerned with the problem of finding out in what manner the Noether decomposition theorems could be extended to a residuated lattice in which the chain condition was weakened and no connection was assumed between irreducibles and primary elements.

* Presented to the Society, March 27, 1937, and April 9, 1938; received by the editors April 21, 1938.

† For a connected account of lattice theory and the literature up to 1937, see Köthe [1]. 
3. We shall use the following terminology and notation. $\subseteq$ is a fixed lattice with elements $a, \cdots, y$ with or without subscripts. Sublattices of $\subseteq$ are denoted by German capitals $\mathfrak{A}, \mathfrak{B}$. The letters $\mathfrak{X}, \mathfrak{Y}, \mathfrak{Z}$ are reserved to denote subsets of $\subseteq$ which are not necessarily sublattices. We write $x \varepsilon \mathfrak{X}$ for "the set $\mathfrak{X}$ contains the element $x$." The expressions $x \supset y$ or $y \subset x, x \ngtr y$ denote, as usual, $x$ divides $y, x$ does not divide $y$. We write $x=y$ if $x \supset y$ and $y \supset x$ (Ore [1], p. 42) and $x>y$ or $y<x$ for $x$ covers $y$ (Birkhoff [1]). We use $(x, y)$ and $[x, y]$ for union and cross-cut. If the unit and null elements exist, we denote them by $i$ and $z$, respectively. Elements covered by $i$ are called divisor-free. If $(a, b)=i, a$ and $b$ are said to be co-prime. If every pair of distinct elements of a set $\mathfrak{X}$ are co-prime, the set is said to be co-prime. An element $n$ of $\mathfrak{S}$ is called a node if either $x \supset n$ or $n \supset x$ for every $x$ of $\mathfrak{S}$. A sublattice $\mathfrak{A}$ is said to be dense over $\mathfrak{S}$ if $a_{1}, a_{2} \varepsilon \mathfrak{A}$ and $a_{1} \supset x \supset a_{2}$ imply $\mathfrak{A}$ contains $x$. If every set of elements finite or infinite of $\subseteq$ has a cross-cut (union), $\subseteq$ is said to be completely closed relative to cross-cut (union). Two properties $P$ and $Q$ which $\subseteq$ may possess are said to be completely independent if there exist instances of lattices in which both $P$ and $Q$ hold, neither holds, $P$ holds but not $Q, Q$ holds but not $P$.

We shall find it convenient to use the following conditions for a distributive lattice either of which is equivalent to the usual formulation:

(i) $b \supset[a, c]$ implies $b=[(b, a),(b, c)]$.

(ii) $(a, c) \supset b$ implies $b=([b, a]$, $[b, c])$.

\section{RESIDUATIONS AND MULTIPLICATIONS}

4. Assume that $\subseteq$ contains $i$. A well-defined one-valued binary operation $x: y$ is called a residuation over $\subseteq$ if the following conditions are satisfied:

$\mathrm{R} 1$. If $\mathfrak{S}$ contains $a, b$, then $\mathfrak{S}$ contains $a: b$.

$\mathrm{R} 2 . a: b=i$ if and only if $a \supset b$.

R 3. $a \supset b$ implies that $a: c>b: c$ and $c: b>c: a$.

R 4. $(a: b): c=(a: c): b$.

R 5. $[a, b]: c=[a: c, b: c]$.

R 6. $c:(a, b)=[c: a, c: b]$.

We postpone the consideration of the dual residuation for our second paper.

A well defined binary operation $x \cdot y$ (or $x y$ ) is called a multiplication over $\subseteq$ if the following conditions are satisfied:

M 1. If $\mathfrak{\subseteq}$ contains $a, b$, then $\subseteq$ contains $a \cdot b$.

M 2. If $a=b$, then $a \cdot c=b \cdot c$.

M 3. $a \cdot b=b \cdot a$. 
M 4. $(a \cdot b) \cdot c=a \cdot(b \cdot c)$.

M 5. If $\subseteq$ contains $i$, then $a \cdot i=a$.

M 6. $a \cdot(b, c)=(a \cdot b, a \cdot c)$.

It may be shown (Ward [1]) that a residuation exists satisfying $R$ 1-R 6 if a multiplication over $\subseteq$ exists satisfying $M 1-M 6$ and the following condition:

M 7. $\subseteq$ is completely closed with respect to union, and the product of the unions of any two sets of elements of $\subseteq$ is the union of the products of all pairs of elements in the sets.

This residual $a: b$, satisfying $\mathrm{R} 1-\mathrm{R} 6$, is defined as follows:

Definition 4.1. (i) $a \supset(a: b) b$; (ii) if $a \supset x b$, then $a: b>x$.

If we take for $x \cdot y$ the cross-cut $[x, y]$, then conditions M 1-M 6 are all satisfied provided that $\subseteq$ is distributive. If $\mathbf{M} 7 \mathrm{holds}$, the lattice is said to be completely distributive with respect to union. Hence (Ward [2]) every completely distributive lattice may be residuated in at least one way.

Another condition* insuring the existence of a residual is the following:

M 8. For any two elements $a, b$ of $\mathfrak{S}$, the ascending chain condition holds in the set $\mathfrak{X}$ of all $x$ such that $a \supset x b$.

M 8 insures the existence of a union of the set $\mathfrak{X}$ expressible as the union of a finite number of elements of $\mathfrak{X}$ (Ore [1], §2). This union is the required residual.

We list for reference the more important properties of residuation and multiplication (Ward [1], Dilworth [1]):

(4.1) $a: b \supset a$.

(4.2) $a:(a: b) \supset(a, b)$.

(4.3) $(a: b): c=a:(b c)$.

(4.4) $[a, b]: b=a: b$.

(4.5) $a:(a, b)=a: b$.

(4.51) $c:[a, b] \supset(c: a, c: b)$.

(4.6) If $a: b=a$, then $a \supset b x$

implies $a \supset x$.
(4.7) If $r=a: b$ and $s=a: r$, then

$$
r=a: s \text {. }
$$

(4.71) $a \supset b$ implies $a c \supset b c$.

(4.8) $[a, b] \supset a b \supset[a, b](a, b)$.

(4.81) $a b: a \supset b$.

(4.9) $\quad a=b c$ implies $b \supset a$.

(4.10) $(a, b)=$ i implies $a b=[a, b]$ and $(a, b c)=(a, c)$.

$$
\text { (4.11) }(a, b): c \supset(a: c, b: c) \text {. }
$$

(4.12) In any chain of powers $a, a^{2}, a^{3}, \ldots$ either all elements are distinct or all are equal from a certain point on.

* This axiom is equivalent to the ascending chain condition, as may be seen on taking $a=b=i$. We state it in this manner to emphasize the analogy with $R 8$ which is not equivalent to the descending chain condition. 
5. We shall now exhibit a remarkable reciprocity between the operations of residuation and multiplication.

THEOREM 5.1. If a residuation $x: y$ exists in $\mathfrak{S}$ satisfying conditions $\mathrm{R} 1-$ $\mathrm{R} 6$, and if either of the conditions $\mathrm{R} 7$ or $\mathrm{R} 8$ below holds, then a multiplication $x \cdot y$ exists in 5 satisfying $\mathbf{M} 1-\mathbf{M} 6$.

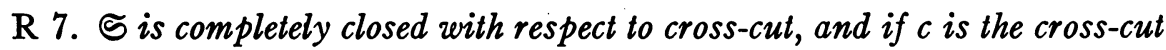
of $a$ set $\mathfrak{X}$, then the cross-cut of the set of all $a: x$, where $a \varepsilon \mathfrak{S}, x \in \mathfrak{X}$, equals $a: c$.

$\mathrm{R} 8$. For any two elements $a, b$ of 5 the descending chain condition holds in the set $\mathfrak{Y}$ of all elements $y$ such that $y: a \supset b$.

$R 8$ is satisfied in many important instances where $R 7$ does not hold and where the descending chain condition does not hold; for example, in polynomial ideal theory and the classical ideal theory of algebraic rings.

The proof is as follows. Define the "product" $a \cdot b$ of any two elements $a$ and $b$ of $\mathfrak{S}$ :

Definition 5.1. (i) $a \cdot b: a \supset b$; (ii) if $y: a \supset b$, then $y>a \cdot b$.

Postulate $\mathrm{M} 1$ is satisfied. For the set $\mathfrak{V}$ of all $y$ such that $y: a \supset b$ is nonempty, since it includes $b$ by (4.1). If $\mathrm{R} 7$ holds, $\mathfrak{V}$ has a cross-cut $p=a \cdot b$ satisfying Definition 5.1 , (ii), and the cross-cut [ $[\mathfrak{y}: a]$ equals $p: a$. Definition 5.1, (i) is therefore satisfied with $p=a \cdot b$ by the definition of cross-cut.

If $R \mathbf{~ h o l d s , ~ t h e n ~} \mathfrak{V}$ again has a cross-cut $p$ representable as the cross-cut of a finite number of $y, p=\left[y_{1}, \cdots, y_{k}\right]$. Thus Definition 5.1, (ii) is satisfied, and Definition 5.1, (i) is satisfied by $R 5$.

Postulate M 2 is satisfied. For by $\mathrm{R} 3, a=b$ implies $a \supset b$ implies $a \cdot c: b>a \cdot c: a$. Hence by Definition 5.1, (i), $a \cdot c: b>c$ so that by Definition 5.1, (ii); $a \cdot c \supset b \cdot c$. Similarly $a=b$ implies $b \cdot c,>a \cdot c$, so that $\mathbf{M} 2$ follows.

Postulate M 3 is satisfied. For $b \cdot a$ exists, and by Definition 5.1, if $y: b>a$, then $y \supset b \cdot a$. Now by R 4, Definition 5.1, (i) and R 2, $(a \cdot b: b): a=(a \cdot b: a): b=i$. Hence by R $2, a \cdot b: b>a$. Hence $a \cdot b>b \cdot a$. Similarly, $b \cdot a \supset a \cdot b, a \cdot b=b \cdot a$. Condition $\mathrm{R} 4$ is thus seen to insure that multiplication is commutative.

Postulate M 4 is satisfied. For by Definition 5.1, (i), $\{a \cdot(c \cdot b)\}: a \supset c \cdot b$. Hence $\{\{a \cdot(c \cdot b)\}: a\}: c>c \cdot b: c$ by R 3. But $c \cdot b: c \supset b$ by Definition 5.1, (i). Therefore $\{\{a \cdot(c \cdot b)\}: a\}: c \supset b$ or by $\mathbf{R} 4,\{\{a \cdot(c \cdot b)\}: c\}: a \supset b$. Hence $\{a(c b): c\} \supset a b$ and $a(c b) \supset c(a b)$ by Definition 5.1, (ii). Interchanging $a$ and $c$, $c(a b) \supset a(c b)$. Hence $a(c b)=c(a b)$, or by $\mathrm{M} 3$ and $\mathrm{M} 2,(a b) c=a(b c)$.

Postulate M 5 is satisfied. For by R 2, $a: a \supset i$. Hence $a \supset a i$ by Definition 5.1, (ii). Now $i a: i>a$ by Definition 5.1, (i). But by (4.10) and M 3, ia:i=ia $=a i$. Hence $a i \supset a, a=a i$.

Postulate M 6 is satisfied. For since $(b, c) \supset b, a(b, c) \supset a b$ by (4.71). Similarly $a(b, c) \supset a c$. Hence $a(b, c) \supset(a b, a c)$. Next $(a b, a c): a \supset a b: a \supset b$ by R 3 
and (4.81). Similarly $(a b, a c): a \supset c$. Hence $(a b, a c): a \supset(b, c)$. Therefore by Definition 5.1, (ii), ( $a b, a c) \supset a(b, c)$ giving M 6. This completes the proof.

DefinITION 5.2. (i) $a \supset(a \circ b) b$; (ii) if $a \supset x b$, then $a \circ b \supset x$.

The following theorem further illustrates the reciprocity between multiplication and residuation:

THEOREM 5.2. If $a \circ b$ is defined as above, where the multiplication $x y$ is defined by Definition 5.1, then $a \circ b=a: b$.

For since $a: b \supset a: b$, we have $a \supset(a: b) b$ by Definition 5.1 , (ii). Therefore by Definition 5.2, (ii), $a \circ b>a: b$. Now $a \supset(a \circ b) b$ by Definition 5.2, (i). Therefore by R 3, $a: b \supset\{(a \circ b) b: b\}$. But by $\mathrm{M} 3$ and Definition 5.1, (ii), $(a \circ b) b: b \supset a \circ b$. Hence $a: b \supset a \circ b, a: b=a \circ b$.

Hereafter when we speak of a "residuated lattice," we shall mean a lattice in which both a residuation and its associated multiplication are defined satisfying M 1-M 6, R 1-R 6 and the conditions of Definitions 5.1 and 5.2.

6. We may prove by simple examples the following theorem:

THEOREM 6.1. The Dedekind modular condition and the existence of a residual or a multiplication are completely independent properties of a lattice.

It is important to observe that a given lattice may usually be residuated in several different ways. To give a simple example, consider the lattice of four elements $i>a>b>z$. The tables for $x: y$ and $x \cdot y$ are as follows:

\begin{tabular}{c|cccc}
$x: y$ & $i$ & $a$ & $b$ & $z$ \\
\hline$i$ & $i$ & $i$ & $i$ & $i$ \\
$a$ & $a$ & $i$ & $i$ & $i$ \\
$b$ & $b$ & $*$ & $i$ & $i$ \\
$z$ & $z$ & $*$ & $*$ & $i$
\end{tabular}

\begin{tabular}{c|cccc}
$x \cdot y$ & $i$ & $a$ & $b$ & $z$ \\
\hline$i$ & $i$ & $a$ & $b$ & $z$ \\
$a$ & $a$ & $*$ & $*$ & $z$ \\
$b$ & $b$ & $*$ & $*$ & $z$ \\
$z$ & $z$ & $z$ & $z$ & $z$
\end{tabular}

A brief analysis discloses that the combinations denoted by stars may be determined in six ways so as to satisfy $R$ 1-R 8, M 1-M 8:

\begin{tabular}{c|cccccc} 
& I & II & III & IV & V & VI \\
\hline$b: a$ & $a$ & $a$ & $b$ & $a$ & $b$ & $b$ \\
$z: a$ & $a$ & $b$ & $b$ & $z$ & $z$ & $z$ \\
$z: b$ & $a$ & $a$ & $a$ & $z$ & $b$ & $z$ \\
$a \cdot a$ & $z$ & $b$ & $a$ & $b$ & $a$ & $a$ \\
$a \cdot b$ & $z$ & $z$ & $z$ & $b$ & $b$ & $b$ \\
$b \cdot b$ & $z$ & $z$ & $z$ & $b$ & $z$ & $b$
\end{tabular}


Cases II and VI are illustrated in the lattice of the ring of integers modulo 8. Here $i$ is the set of residue classes $\{1,3,5,7\}, a$ is $\{2,6\}, b$ is $\{4\}$, and $z$ is $\{8\}$. Case.II ensues on taking for $x \cdot y$ multiplication modulo 8 , and case VI on taking for $x \cdot y$ the L.C.M. operation.

The only other lattice of order four is $i, a, b, z$ with $(a, b)=i,[a, b]=z$. This lattice may be residuated in only one way, an illustration of a general theorem on the residuation of Boolean algebras which we prove later.

\section{CoNDITIONS FOR RESIDUATION}

7. In this division of the paper we shall give various sufficient conditions and necessary conditions for the existence of a residuation in a given lattice.

THEOREM 7.1. A necessary condition that a lattice $\subseteq$ can be residuated is that any co-prime set of elements of $\mathfrak{S}, a_{1}, a_{2}, \cdots, a_{r}$ generates $a$ Boolean algebra $\mathfrak{B}$ of order $2^{r}$.

This condition is not sufficient for a residuation to exist. It is satisfied, for example, in Dedekind's free modular lattice on three elements of order twenty-eight (Dedekind [1], Birkhoff [1], Ore [1]) which we shall prove later cannot be residuated.

Let $a_{1}, a_{2}, \cdots, a_{r}$ be a co-prime set so that

$$
\left(a_{u}, a_{v}\right)=i, \quad u, v=1, \cdots, r ; u \neq v .
$$

The set will remain co-prime if we adjoin $i$ to it. We shall suppose that this has been done, and for definiteness choose our notation so that $a_{1}=i$.

Form from the set of $a$ 's the "ray" $\Pi$ of $2^{r}$ formally distinct cross-cuts:

$$
u=\left[a_{u_{1}}, a_{u_{2}}, \cdots, a_{u_{L}}\right], \quad 1 \leqq u_{1}<u_{2}<\cdots<u_{L} \leqq r ; 1 \leqq L \leqq r .
$$

We call the $a_{u}$ the constituents of $u$. The ray $\Pi$ is obviously closed under crosscut. We shall show that $\Pi$ is the Boolean algebra required.

LEMMA 7.1. If $x$ is any element of $\subseteq$, then

$$
\left(x,\left[a_{u}, a_{v}\right]\right)=\left[\left(x, a_{u}\right),\left(x, a_{v}\right)\right] .
$$

This result is trivial if $u=v$. But if $u \neq v,\left(a_{u}, a_{v}\right)=i$. Hence $\left(\left(x, a_{u}\right),\left(x, a_{v}\right)\right)$ $=i$. Therefore by (4.10) and M 6,

$$
\begin{aligned}
{\left[\left(x, a_{u}\right),\left(x, a_{v}\right)\right] } & =\left(x, a_{u}\right)\left(x, a_{v}\right)=\left(x^{2}, x a_{v}, a_{u} x, a_{u} a_{v}\right) \\
& =\left(x^{2}, x\left(a_{u}, a_{v}\right), a_{u} a_{v}\right)=\left(x, a_{u} a_{v}\right)=\left(x,\left[a_{u}, a_{v}\right]\right)
\end{aligned}
$$

by $\mathrm{M} 3$ and $\mathrm{M} 6$.

The following two corollaries of this lemma may be proved by induction:

LEMMA 7.2. If $u=\left[a_{u_{1}}, \cdots, a_{u_{L}}\right]$, then $(x, u)=\left[\left(x, a_{u_{1}}\right), \cdots,\left(x, a_{u_{L}}\right)\right]$. 
LEMMA 7.3. If $u=\left[a_{u_{1}}, \cdots, a_{u_{L}}\right]$ and $v=\left[a_{v_{1}}, \cdots, a_{v_{M}}\right]$, then

$$
(u, v)=\left[\left(a_{u_{1}}, a_{v_{1}}\right), \cdots,\left(a_{u}, a_{v}\right), \cdots,\left(a_{u_{L}}, a_{v_{M}}\right)\right] .
$$

LEMMA 7.4. If $x$ is any element of $\mathfrak{S}$ and if $(x, b)=(x, c)=i$, then

$$
(x,[b, c])=[(x, b),(x, c)] .
$$

It suffices to show that $(x,[b, c])=i$. But $(x,[b, c]), \supset(x b c)=(x, b x, b c)$ (by $(4.10))=(x, b(x, c))=(x, b)=i$.

We return to the proof of our theorem. The ray $I$ is a lattice. For by Lemma 7.3 and (7.1) it is closed under union. The lattice is of order $2^{r}$. It suffices to show that if $u=v$, the constituents of $u$ and $v$ are identical. But if $u=v, a_{u} \supset v$. Hence by Lemma 7.2,

$$
a_{u}=\left(a_{u}, v\right)=\left[\left(a_{u}, a_{v_{1}}\right), \cdots,\left(a_{u}, a_{v_{M}}\right)\right] .
$$

Since $\left(a_{u}, a_{v}\right)=a_{u}$ or $i, a_{u}$ must be a constituent of $v$. Thus every constituent of $u$ is a constituent of $v$. Similarly every constituent of $v$ is a constituent of $u$, so that $u$ and $v$ are not formally distinct.

The lattice is distributive. For by Lemma 7.3 , if $w=\left[a_{w_{1}}, \cdots, a_{w_{N}}\right]$, then

$$
\begin{aligned}
(w,[u, v]) & =\left[\cdots,\left(a_{w},[u, v]\right), \cdots\right] \\
& =\left[\cdots,\left[\left(a_{w}, u\right),\left(a_{w}, v\right)\right], \cdots\right] \\
& =\left[\left[\cdots,\left(a_{w}, u\right), \cdots\right],\left[\cdots,\left(a_{w}, v\right), \cdots\right]\right] \\
& =[(w, u),(w, v)],
\end{aligned}
$$

by Lemma 7.2.

The lattice is complemented. For we assign to the element $u$ the complement

$$
u^{\prime}=\left[a_{u^{\prime} L+1}, \cdots, a_{u_{r}^{\prime}}\right]
$$

where $u_{L+1}^{\prime}, \cdots, u_{r}^{\prime}$ is the selection complementary to $u_{1}, \cdots, u_{L}$ from $1,2, \cdots, r$. Then $\left[u, u^{\prime}\right]=\left[a_{1}, a_{2}, \cdots, a_{r}\right]$, the null element of the lattice $\mathfrak{B}$, and $\left(u, u^{\prime}\right)=i$ by Lemma 7.3. Hence $\mathfrak{B}$ is a complemented distributive lattice and thus a Boolean algebra.

TheOREM 7.2. If $a_{1}, \cdots, a_{r}$ is a co-prime set of divisor-free elements of a residuated lattice $\mathfrak{S}$, then the Boolean algebra $\mathfrak{B}$ which they generate is dense over S.

For if $u$ lies in $\mathfrak{B}$ and $x \supset u$, then $x=\left[\left(x, a_{u_{1}}\right), \cdots,\left(x, a_{u_{L}}\right)\right]$ by Lemma 7.2. Since $\left(x, a_{u}\right)=i$ or $a_{u}$, the result follows.

This theorem is quite useful in examining finite lattices to see whether or not they can be residuated. We have also found the following exclusion principle useful in this connection. The proof (which we omit) follows from Lemma 7.4. 
ThEOREM 7.3. EXCLUSION PRINCIPLE. Let $a, b, c$, and $d$ be any four elements of a residuated lattice $\mathbb{S}$ with an ascending chain condition such that $c \supset a, c \neq i ;(b, c)=i, d>a, d>b$. Then if $m=[b, c]$ we must have $[a, b]=m$ and $a>m, b>m$. Furthermore $a$ and $b$ are the only elements covered by $d$ and covering $m$ in the lattice.

In schematic form (Klein [1], Birkhoff [2]) the lattice must have the following structure, where the dotted lines indicate that the configuration of the remaining lattice parts is irrelevant.

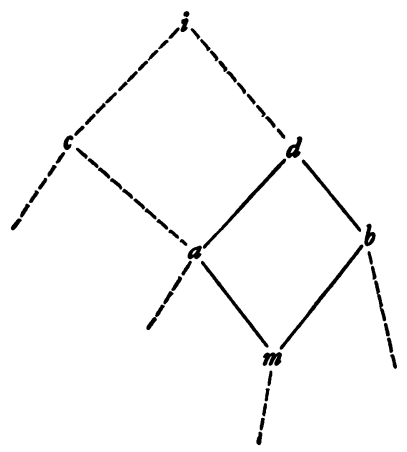

As a simple application, if the reader will diagram the lattice of order nine on three elements $b, c$, and $f$ where $c \supset f$ (Dedekind [1]) and take $a=[c,(f, b)], d=(f, b)$, he will see that this lattice cannot be residuated.

THEOREM 7.31. The only complemented lattices which can be residuated are Boolean algebras.

Since by hypothesis the lattice is complemented, it is sufficient to show that it is distributive. We need the following lemma:

LEMMA 7.5. If $(b, c)=i$ and $a \supset[b, c]$, then $(a: b, a: c)=i$.

For we have

$$
\begin{aligned}
(a: b, a: c) & =(a: b, a: c):(b, c)=[(a: b, a: c): b,(a: b, a: c): c] \\
& \supset[(a: c): b,(a: b): c]=a: c b=a:[c, b]=i .
\end{aligned}
$$

A complement $a^{\prime}$ of $a$ is defined by the following conditions:

Definition 7.1. $\left(a, a^{\prime}\right)=i,\left[a, a^{\prime}\right]=z$, where $z$ is the null element of $\mathfrak{S}$.

Let $a, b, c$ be any three elements of $\mathfrak{S}$ and assume that

$$
a \supset[b, c] \text {. }
$$

Let $u=[(a, b),(a, c)]$ and $v=\left([b, c], a^{\prime}\right)$. It suffices to show that (i) im- 
plies $u=a$. We have trivially $u \supset a$ and $v \supset a^{\prime}$. Hence $(u, v)=i$ by Definition 7.1 so that $u v=[u, v]$. Hence $b: u v=b:[u, v] \supset(b: u, b: v)$ by (4.51). Now $b: u \supset b: a$ by $\mathrm{R} 3$ and $b: v=\left[b:[b, c], b: a^{\prime}\right]=b: a^{\prime}$. Hence $b: u v \supset\left(b: a, b: a^{\prime}\right)$. But by Definition 7.1, $\left(a, a^{\prime}\right)=i$ and $b \supset\left[a, a^{\prime}\right]$. Hence by Lemma 7.5, $\left(b: a, b: a^{\prime}\right)=i$ so that $b \supset u v$. Similarly $c \supset u v$ so that $[b, c] \supset u v$, or by (i), $a \supset u v, a: v \supset u$. But $a: v=\left[a:[b, c], a: a^{\prime}\right]=a: a^{\prime}=a$ by (i) and Definition 7.1. Hence $a \supset u$ so that $a=u$.

COROLlaRy. The only projective geometries (Birkhoff [3]) which can be residuated are Boolean algebras.

In case the ascending chain condition holds in $\mathfrak{S}$, one can give a much shorter proof by showing that each element may be represented as a cross-cut of a finite number of divisor-free elements and appealing to Theorem 7.1.

THEOREM 7.4. The only multiplication which can be defined over a Boolean algebra is the cross-cut operation.

In view of our reciprocity theorems it suffices to show that only one residual is definable. One of us has shown elsewhere (Dilworth [1]) that $a \vee b^{\prime}$ is a residuation in a Boolean algebra. Suppose that $a: b$ were another. Then

$$
\begin{aligned}
& (a: b):\left(a \vee b^{\prime}\right)=(a: b): b^{\prime}=a: b b^{\prime}=i \\
& \left(a \vee b^{\prime}\right):(a: b) \supset\{a:(a: b)\} \vee\left\{b^{\prime}:(a: b)\right\} \supset b \vee b^{\prime}=i .
\end{aligned}
$$

Hence $a: b=a \vee b^{\prime}$.

An interesting consequence of Theorem 7.4 is the following corollary:

COROLLARY. In the ring of integers modulo a square-free integer, the operations of multiplication and L.C.M. are identical.

8. We consider in this section some sufficient conditions for residuation. We have the following theorem:

THEOREM 8.1. Every lattice in which only one divisor-free element exists can residuated in at least one way.

Let $d$ be the single divisor-free element. We define the residual $a: b$ by the conditions:
(i) $a: i=a$;
(ii) $a: b=i$ if $a \supset b$;
(iii) $a: b=d$ if $a \ngtr b, b \neq i$.

Then postulates $R 1$ and $R 2$ are obviously satisfied.

$\mathrm{R} 3$ is satisfied. For assume $a \supset b$. Then $a: c$ always divides $b: c$ except possibly when $b: c=i$. But then $b \supset c$; so $a \supset c, a: c=i$. Similarly $c: b \supset c: a$.

$\mathrm{R} 4$ is satisfied. For R 4 obviously holds if $a, b$, or $c$ equals $i$. If $a \supset b, a \neq i$, 
then $a: c>a \supset b$; so $(a: c): b=(a: b): c=i$. If $a: b>c$ but $a \$ b, b \neq i, a \gg c, c \neq i$, then $a: b=a: c=d$, whence $(a: b): c=d: c=i=d: b=(a: c): b$. If $a \gg b, a \gg c$, $a: b \$ c, a: c \$ b$, then $b$ or $c=i$.

$\mathrm{R} 5$ is satisfied. For if $c=i, \mathrm{R} 5$ is trivial. If $a \supset c, b \supset c$, then $[a, b] \supset c$ and $\mathrm{R} 5$ obviously holds. If $a \ngtr c, c \neq i$, then $[a, b] \ngtr c$ and $[a, b]: c=d=[a: c, b: c]$. Hence R 5 holds in general.

In exactly the same way we show that $\mathrm{R} 6$ is satisfied.

F. Klein has shown (Klein [1]) that the modular or distributive properties of a lattice built up of sublattices connected by nodes ("Schnurstellen") depend upon the modular or distributive properties of the sublattices. We prove a similar result for residuation.

THEOREM 8.2. A lattice built up out of a set of residuated lattices connected into a chain by nodes can be residuated.

It will suffice to prove the theorem for the case of two lattices connected by a node.

Let $\subseteq$ be composed of two lattices $\widetilde{S}_{1}$ and $\widetilde{S}_{2}$ connected by a node, so that $x_{1} \varepsilon \Im_{1}$ and $x_{2} \varepsilon \Im_{2}$ imply $x_{1} \supset x_{2}$. Let $i$ be the unit element of $\Im_{1}$. We shall consider the nodal element as belonging to $\Im_{1}$, and let $x: y$ denote the residuation in $\Im_{1}, x \circ y$, the residuation in $\Im_{2}$ when the nodal element is replaced by $i$.

We now define a residual in $\subseteq$ by the conditions:

$a: b=a: b$ if $a, b \varepsilon \mathfrak{S}_{1}, a: b=a \circ b$ if $a, b \varepsilon \Im_{2}$,

$a: b=i$ if $a \varepsilon \Im_{1}, b \varepsilon \Im_{2}, a: b=a$ if $a \varepsilon \Im_{2}, b \varepsilon \Im_{1}$.

Then postulates R 1, R 2, and R 3 are obviously satisfied.

Postulate R 4 is satisfied. For clearly $a: c \supset a$. Hence if $a \supset b$, then $(a: b): c=(a: c): b$ by R 3. Also if $a \varepsilon \mathfrak{S}_{1}$, then $(a: b): c=(a: c): b$. Suppose that $a \varepsilon \Im_{2}$. Then if $b \varepsilon \Im_{2}, c \varepsilon \Im_{2}$, we have $(a: b): c=(a: c): b$. Similarly if $b \varepsilon \Im_{1}$, $c \varepsilon \dot{\mathfrak{S}}_{1}$, then $(a: b): c=(a: c): b$. Finally if $b \varepsilon \mathfrak{S}_{1}, c \varepsilon \mathfrak{S}_{2}$, then $(a: b): c=a \circ c$ $=(a: c): b$.

Postulate R 5 is satisfied. For R 5 is trivial if $a, b$, or $c=i$. If $a \supset b$, R 5 follows from R 3. If $a, b \varepsilon \Im_{1}$ or $a, b \varepsilon \Im_{2}, \mathrm{R} 5$ holds since it holds in $\mathfrak{S}_{1}$ and $\mathfrak{S}_{2}$.

In a similar manner one can show that $R 6$ is satisfied, and the proof is complete.

By the direct product (Birkhoff [4]) $\subseteq$ of the lattices $\Im_{1}, \cdots, \Im_{n}$ we mean the set of vectors $a=\left\{a_{1}, \cdots, a_{n}\right\},\left(a_{i} \varepsilon \Im_{i}\right)$, where the operations are defined by

$$
\begin{aligned}
& {[a, b]=\left\{\left[a_{1}, b_{1}\right], \cdots,\left[a_{n}, b_{n}\right]\right\},} \\
& (a, b)=\left\{\left(a_{1}, b_{1}\right), \cdots,\left(a_{n}, b_{n}\right)\right\},
\end{aligned}
$$

and $a \supset b$ if and only if $a_{i} \supset b_{i},(i=1, \cdots, n)$. 
If the $\mathfrak{S}_{i}$ are residuated lattices, then $\mathfrak{S}$ can be residuated, since we may define $a: b$ to be $\left\{a_{1}: b_{1}, \cdots, a_{n}: b_{n}\right\}$.

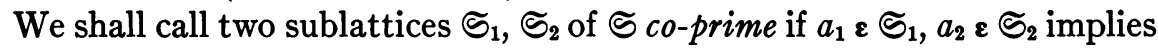
that $\left(a_{1}, a_{2}\right)=i$. The sublattices $\mathfrak{S}_{1}, \cdots, \mathfrak{S}_{n}$ will be called a co-prime set if they are co-prime in pairs.

We note that if $\subseteq$ is the direct product of the sublattices $\mathfrak{S}_{1}, \cdots, \mathfrak{S}_{n}$ with unit elements, then $\mathfrak{S}$ contains sublattices $\mathfrak{S}_{1}^{\prime}, \ldots, \mathfrak{S}_{n}^{\prime}$ simply isomorphic to $\mathfrak{S}_{1}, \cdots, \mathfrak{S}_{n}$ and such that $\mathfrak{S}_{1}^{\prime}, \cdots, \mathfrak{S}_{n}^{\prime}$ is a co-prime set. Birkhoff (Birkhoff [4]) has defined sublattices $\mathfrak{S}_{1}, \cdots, \mathfrak{S}_{n}$ to be "strongly" coprime if each $\mathfrak{S}_{i}$ is co-prime to the lattice generated by the remaining lattices. Clearly strong co-primeness implies co-primeness in the ordinary sense. Moreever if $\subseteq$ is residuated, Lemma 7.4 shows that co-primeness implies strong co-primeness, so that for residuated lattices the notions are identical. We now prove a converse result.

THEOREM 8.3. Let $\mathfrak{S}_{1}, \mathfrak{S}_{2}, \cdots, \mathfrak{S}_{n}$ be a set of co-prime sublattices of a residuated lattice $\mathfrak{S}$ such that each element of $\mathfrak{S}$ can be expressed as a cross-cut of elements of $\mathfrak{S}_{1}, \cdots, \mathfrak{S}_{n}$. Then $\mathfrak{S}_{\text {is }}$ the direct product of $\mathfrak{S}_{1}, \cdots, \mathfrak{S}_{n}$, and each $\mathfrak{S}_{i}$ can be residuated.

Let $a=\left[a_{1}, a_{2}, \cdots, a_{n}\right],\left(a_{i} \varepsilon \mathfrak{S}_{i}\right), b=\left[b_{1}, b_{2}, \cdots, b_{n}\right],\left(b_{i} \varepsilon \mathfrak{S}_{i}\right)$. Then $[a, b]=\left[\left[a_{1}, b_{1}\right], \cdots,\left[a_{n}, b_{n}\right]\right]$. Furthermore

$$
(a, b)=\left[\left(a_{1}, b_{1}\right), \cdots,\left(a_{n}, b_{n}\right)\right] .
$$

For by Lemma 7.2,

$$
\begin{aligned}
(a, b) & =\left(a,\left[b_{1}, \cdots, b_{n}\right]\right)=\left[\left(a, b_{1}\right), \cdots,\left(a, b_{n}\right)\right] \\
& =\left[\cdots,\left(a_{j}, b_{k}\right), \cdots\right]=\left[\left(a_{1}, b_{1}\right), \cdots,\left(a_{n}, b_{n}\right)\right],
\end{aligned}
$$

since $\left(a_{j}, b_{k}\right)=i$ if $j \neq k$.

LEMMA 8.1. If $b_{1}, b_{2}, \cdots, b_{n}$ are a co-prime set, then

$$
a:\left[b_{1}, \cdots, b_{n}\right]=\left(\cdots\left(\left(\left(a: b_{1}\right): b_{2}\right): b_{3}\right) \cdots\right): b_{n} .
$$

This result follows by repeated applications of Lemma 7.4, (4.3), and (4.10).

We have now $a: b=a:\left[b_{1}, \cdots, b_{n}\right]=\left(\cdots\left(\left(a: b_{1}\right): b_{2}\right) \cdots\right): b_{n}$ by Lemma 8.1. But

$$
\begin{aligned}
a: b_{i} & =\left[a_{1}, \cdots, a_{n}\right]: b_{i}=\left[a_{1}: b_{i}, \cdots, a_{n}: b_{i}\right] \\
& =\left[a_{1}, \cdots, a_{i-1}, a_{i}: b_{i}, a_{i+1}, \cdots, a_{n}\right] .
\end{aligned}
$$

Hence $a: b=\left[a_{1}: b_{1}, a_{2}: b_{2}, \cdots, a_{n}: b_{n}\right]$.

If $a=b$, then $\left(a_{i},\left[a_{1}, \cdots, a_{n}\right]\right)=\left(a_{i},\left[b_{1}, \cdots, b_{n}\right]\right)$ or $a_{i}=\left(a_{i}, b_{i}\right), a_{i} \supset b_{i}$. 
Similarly $b_{i} \supset a_{i}$. Hence $\mathfrak{S}$ is simply isomorphic with the direct product of the $\mathfrak{S}_{i}$. We note that if $x=\left[x_{1}, \cdots, x_{n}\right],\left(x \supset a_{i}, a_{i} \varepsilon \mathfrak{S}_{i}\right)$, then

$$
x=\left(x, a_{i}\right)=\left(\left[x_{1}, \cdots, x_{n}\right], a_{i}\right)=\left(x_{i}, a_{i}\right) \varepsilon \mathfrak{S}_{i} .
$$

Since $a_{i}: b_{i} \supset a_{i}$, we see that $\mathfrak{S}_{i}$ is closed under residuation, which completes the proof.

We conclude with a theorem of a more special character.

THEOREM 8.4. The free modular lattice of order twenty-eight on three elements cannot be residuated.

We shall use Dedekind's original notation for the elements of this lattice in the proof of the theorem (Dedekind [1]). Assume that a residuation $x: y$ exists. Then $\mathfrak{b}^{\prime \prime \prime \prime}$ is the unit element. Hence $\mathfrak{a}_{0}: \mathfrak{b}^{\prime}=\mathfrak{b}_{0}: \mathfrak{b}^{\prime}=\mathfrak{c}_{0}: \mathfrak{b}^{\prime}=\mathfrak{h} \neq \mathfrak{b}^{\prime \prime \prime \prime}$. Now $\mathfrak{a}_{0}=\left[\mathfrak{a}^{\prime}, \mathfrak{a}^{\prime \prime \prime}\right]$. Hence $\mathfrak{a}_{0}: \mathfrak{a}^{\prime \prime \prime}=\mathfrak{a}^{\prime}: \mathfrak{a}^{\prime \prime \prime} \supset \mathfrak{a}^{\prime}$. But $\mathfrak{a}^{\prime \prime \prime} \supset \mathfrak{b}^{\prime}$. Therefore $\mathfrak{a}_{0}: \mathfrak{b}^{\prime} \supset \mathfrak{a}_{0}: \mathfrak{a}^{\prime \prime \prime}$ or $\mathfrak{h} \supset \mathfrak{a}^{\prime}$. Similarly, $\mathfrak{h} \supset \mathfrak{b}^{\prime}, \mathfrak{h} \supset \mathfrak{c}^{\prime}$. Hence $\mathfrak{h} \supset\left(\mathfrak{a}^{\prime}, \mathfrak{b}^{\prime}, \mathfrak{c}^{\prime}\right)$ or $\mathfrak{h} \supset \mathfrak{d}^{\prime \prime \prime \prime}, \mathfrak{h}=\mathfrak{d}^{\prime \prime \prime \prime \prime}$ giving a contradiction.

It may be observed that the "exclusion principle" of Theorem 7.3 cannot be applied to prove this theorem.

\section{NOETHER LATTICES*}

9. Consider any residuated lattice $\mathfrak{S}$. An element $c$ of $\mathfrak{S}$ is irreducible if in every decomposition $c=[g, f]$ into a cross-cut of two elements of $\mathfrak{S}$, either $g=c$ or $f=c$. An element $p$ is a prime if $p \supset a b$ implies $p \supset a$ or $p \supset b$, and primary if $p \supset a b, p \triangleright a$ implies $p \supset b^{\circ}$ for some integer $s$. The irreducible elements are thus determined by an intrinsic lattice property, while the primes and primary elements depend upon the particular multiplication introduced into the lattice.

We propose here the name "Noether lattice" for any lattice $\subseteq$ satisfying the following three conditions:

$\mathrm{N}$ 1. The lattice $\subseteq$ may be residuated.

$\mathrm{N}$ 2. The ascending chain condition holds in 5 .

$\mathrm{N}$ 3. Every irreducible element of $\subseteq$ is primary.

By $\mathrm{N} 1$ we mean that $\subseteq$ is closed under operations $x: y, x y$ having the properties R 1-R 6, M 1-M 6 and connected by the relationships expressed

* Our definition differs from that in Ward and Dilworth [1]. We have found that some of the results stated in $\$ 4$ of this paper are in error. In postulate $\mathrm{D} 1$, the exponent $r$ must be replaced by 1 . The condition $a b=[a, b]$ on the idempotent elements of a finite modular lattice is consequently necessary for the truth of $\mathrm{D} 1$ but not sufficient. The postulate $\mathrm{M} 7$ is not a sufficient condition for a Noether lattice as stated in the theorem preceding $M 7$. 
in Definitions 4.1, 5.1. By N 2 we mean (Ore [1]) that every chain of lattice elements $a_{1}<a_{2}<a_{3}<\cdots<i$ terminates.

We choose the name in honor of Emmy Noether because the decomposition theorems first proved by her for the ideals of a commutative ring with chain condition all hold. It is to be observed that we do not assume a modular condition.

The proof that the usual decomposition theorems hold may be made by a mere transcription of the proofs given in van der Waerden [1] into lattice language. With each primary $q$ is associated a prime $p$ with the properties $p \supset q$ and $p \supset b$ implies $q \supset b^{r}$. A cross-cut of primaries is said to be "simple" if no primary in it divides the cross-cut of any of the remaining primaries. Every element not equal to $i$ of a Noether lattice may be represented as a simple cross-cut of a finite number of primaries each of which is associated with a different prime. The primes themselves and the total number of primaries are uniquely determined by the element. We obtain from each such representation a representation as the cross-cut of "isolated components" by grouping together the cross-cuts of primaries whose associated primes divide one another. The isolated components of an element and the corresponding representation as their cross-cut are unique.

As was pointed out by Krull (Krull [1]), the decompositions into relatively prime ("teilerfremd") elements depend merely upon $\mathrm{N} 1$ and $\mathrm{N} 2$. From our standpoint, they are simple consequences of Theorem 7.1 and the chain condition.

We may specialize our lattice still more by the following assumption:

$\mathrm{N} 4$. Every prime of $\subseteq$ is divisor-free.

Then, since we have trivially from $\mathrm{N} 1$ that every divisor-free element is a prime, we easily see that all primaries associated with a given prime form a lattice which we may say "belongs" to this prime.

The lattices belonging to distinct primes have no elements save $i$ in common. Hence the decomposition theorems in this case are merely an instance of Birkhoff's decomposition of a lattice into direct products relative to crosscut (Birkhoff [4]).

10. We shall now give some general properties of any Noether lattice.

THEOREM 10.1. If $a$ and $b$ are any two elements of a Noether lattice, there exists an exponent s such that the following condition holds:

D 1. $a b \supset\left[a, b^{s}\right]$.

Let $a b=\left[q_{1}, \cdots, q_{k}\right]$ be a decomposition of $a b$ into a cross-cut of primaries. Then for each $q_{i}, q_{i} \supset a b$; hence either $q_{i} \supset a$ or $q_{i} \gg a, q_{i} \supset b^{s_{i}}$. With 
a proper choice of notation, we may assume that $q_{i} \supset a,(i=1, \cdots, l), q_{i} \supset b^{s^{i}}$, $(i=l+1, \cdots, k)$. Hence if $s$ is the largest of the $s_{i},\left[q_{1}, \cdots, q_{l}\right] \supset a$, $\left[q_{l+1}, \cdots, q_{k}\right] \supset b^{s}$ giving D 1 .

The following three theorems are immediate corollaries:

THEOREM 10.2. If $b$ is an idempotent element in a Noether lattice, then $[a, b]=a b$ for any other element $a$ of the lattice, and $b[a, c]=[b a, b c]$ for any elements $a$ and $c$.

THEOREM 10.3. In a Noether lattice, every idempotent element is neutral.*

ThEOREM 10.4. In a Noether lattice, the idempotent elements form a distributive lattice. The product of any two idempotent elements is their cross-cut.

It is easy to show that this last mentioned property of idempotent elements holds in any lattice in which multiplication is distributive with respect to cross-cut; for if $a, b$ are idempotent,

$$
\begin{aligned}
a b \supset[a, b](a, b) & =[a(a, b), b(a, b)] \\
& =\left[\left(a^{2}, a b\right),\left(b a, b^{2}\right)\right]=[(a, a b),(b, a b)]=[a, b] .
\end{aligned}
$$

The following lattice of order six illustrates how the definition of a Noether lattice depends upon the type of multiplication introduced. The elements are $i, j, a, b, k$, and $z$ with the coverings $i>j, j>a, j>b ; a>k, b>k$; $k>z$. The lattice is distributive and hence a Noether lattice if multiplication is identified with cross-cut (see $\$ 11$ ). Define an operation $x y$ by $i x=x i=x$; $z x=x z=z ; \quad j^{2}=j, \quad a^{2}=a, \quad b^{2}=b, \quad k^{2}=z ; j a=a j=a ; j b=b j=b ; j k=k j=z ;$ $a b=b a=a k=k a=b k=k b=z$. It may be shown that $x y$ is a multiplication satisfying M 1-M 8. But D 1 does not hold; for $a b=z$ and $[a, b]=k$, while $a$ and $b$ are idempotent. Hence $\mathrm{N} 3$ is false by Theorem 10.1 .

11. We shall next give some sufficient conditions that a lattice be.a Noether lattice.

THEOREM 11.1. Let $\subseteq$ be a residuated lattice with ascending chain condition. Then sufficient co:aditions that $\subseteq$ be a Noether lattice are as follows:

D 1. $a b \supset\left[a, b^{s}\right]$.

D 2. $\subseteq$ is modular.

It suffices to show that $\mathrm{N} 3$ holds. Let $m$ be irreducible, $m \supset a b, m \gg a$. Then if $d=(a, m), d \supset m \supset d b$. Now by $\mathrm{D} 1, d b \supset\left[d, b^{s}\right]$ for some $s$. Hence $d \supset m \supset\left[d, b^{s}\right]$. Therefore by D $2, m=\left[(m, d),\left(m, b^{s}\right)\right]$. Since $m$ is irreducible and $(m, d)=(m, a) \neq m,\left(m, b^{s}\right)=m$. Hence $m \supset b^{s}$ and $m$ is primary.

* Following Ore [1], we call an element $n$ of a lattice "neutral" if $[n,(b, c)]=([n, b],[n, c])$ for every pair of elements $b, c$ of the lattice. 
CoROLlary. Every distributive lattice in which the ascending chain condition holds is a Noether lattice for a suitably defined multiplication.

We take for the multiplication the cross-cut operation. Then M 1-M 6 and M 8 all hold; so $\subseteq$ may be residuated. Since $\subseteq$ is distributive, it is modular. Thus N 1, N 2, and D 2 hold. But D 1 is trivially true. The result now follows from the previous theorem.

We shall next give some conditions enabling us to view the ideal theory of commutative rings from a lattice-theoretic standpoint. It is first necessary to introduce a new concept. Let $\subseteq$ be a residuated lattice.

Definition 11.1. An element $q$ of $\mathfrak{S}$ is principal if $q \supset b$ implies that there exists an element $c$ such that $q c=b$.

Neither $c$ nor $b$ need be principal.

Suppose that $a$ is principal, $a \supset b$. The set 3 of elements $z$ such that $a z=b$ is closed with respect to union. If either postulate $M 7$ or $M 8$ holds, the union $b / a$ of $\mathbb{3}$ has the properties stated in the following definition:

Definition 11.2. $a \cdot(b / a)=b$; if $a x=b$ then $b / a \supset x$.

We call $b / a$ the quotient of $b$ by $a$. It is easily shown (Ward [1]) that if $a$ is principal and $a \supset b$, then the quotient $b / a$ equals the residual $b: a$ of $a$ with respect to $b$.

As a simple consequence, we have the following lemma:

LEMMA 11.1. If $a$ is principal and if $a \supset b$, then $b=(b: a) a$.

We may observe that M 8 always holds if the ascending chain condition holds. Hence Lemma 11.1 is true for all principal elements of a residuated lattice with ascending chain condition. We shall now prove the following fundamental theorem:

THEOREM 11.2. Let $\subseteq$ be a lattice in which the following conditions hold:

$\mathrm{N}$ 1. The lattice Ș may be residuated.

$\mathrm{N}$ 2. The ascending chain condition holds in 5 .

D 2. S is modular.

D 3. Every element of $\mathfrak{S}$ is the union of a finite number of principal elements.

D 4. The principal elements of 5 are closed under multiplication.

Then $\subseteq$ is a Noether lattice.

The instance of ideal theory is obtained by identifying the principal elements of the lattice with the principal ideals or the corresponding ring ele- 
ments. D 3 is then the basis theorem, and D 4 the closure property of ring multiplication.

It suffices to show that every irreducible element is primary, or inversely that every non-primary element is reducible. Let $m$ be non-primary. Then there exist elements $a$ and $b$ of the lattice such that

$$
m \supset a b, m \gg a, m \gg b^{r} \text { any } r \text {. }
$$

We shall show that $m$ is reducible. By D $3, b=\left(b_{1}, b_{2}, \cdots, b_{k}\right)$ where the $b_{i}$ are principal. Then $m>a b_{i}$. For at least one $b_{i}, m \ngtr b_{i}{ }^{r}$ for any $r$. For otherwise, for each $b_{i}$ there exists an exponent $r_{i}$ such that $m \supset b_{i}{ }^{r_{i}}$. Then if $r>r_{1}+r_{2}+\cdots+r_{l}-l$, we have $m>b^{r}$ contrary to hypothesis. Therefore, we may assume that $b$ in (11.1) is principal.

By $\mathrm{N} 2$, the chain $m: b, m: b^{2}, \cdots, m: b^{k}, \cdots$ terminates so that $m: b^{k}=m: b^{k+1}$ for some fixed $k$. Consider the cross-cut $c=\left[(m, a),\left(m, b^{k}\right)\right]$. We have trivially $c \supset m$. Now $\left(m, b^{k}\right) \supset c \supset m$. Hence by D 2 (Ore [1]),

$$
c=\left([c, m],\left[c, b^{k}\right]\right) \text {. }
$$

Now $m \supset[c, m]$. We shall show next that $m \supset\left[c, b^{k}\right]$. By D $4, b^{k}$ is principal, and $b^{k} \supset\left[c, b^{k}\right]$. Hence by Lemma 11.1, $\left[c, b^{k}\right]=\left\{\left[c, b^{k}\right]: b^{k}\right\} b^{k}=\left(c: b^{k}\right) b^{k}$. Also since $(m, a) \supset c, b(m, a) \supset b c$. But $b(m, a)=(b m, b a) \subset m$ by (11.1). Hence $m \supset b c \supset b\left[c, b^{k}\right]$ by (11.2). That is, $m \supset b\left\{\left(c: b^{k}\right) b^{k}\right\}$ or $m: b^{k+1} \supset c: b^{k}$. But $m: b^{k+1}=m: b^{k}$. Hence $m: b^{k} \supset c: b^{k}$ or $m \supset\left(c: b^{k}\right) b^{k}, m \supset\left[c, b^{k}\right]$. It follows therefore that $m \supset c$. Hence $m=c$ or $m=\left[(m, a),\left(m, b^{k}\right)\right]$. But $m \ngtr a, m \ngtr b^{k}$. Hence $(m, a) \neq m,\left(m, b^{k}\right) \neq m$, and $m$ is reducible. This completes the proof.

12. To show the significance of the hypotheses of Theorems 11.1 and 11.2, we shall exhibit various lattices in which not all the hypotheses are satisfied.

We first consider the following lattice $\mathfrak{B}_{1}$; and we define a multiplication $x y$ over $\mathfrak{B}_{1}$ by the following table:

\begin{tabular}{c|cccccc}
$x$ & $i$ & $j$ & $a$ & $b$ & $m$ & $z$ \\
$i$ & $i$ & $j$ & $a$ & $b$ & $m$ & $z$ \\
$j$ & $j$ & $a$ & $a$ & $z$ & $z$ & $z$ \\
$a$ & $a$ & $a$ & $a$ & $z$ & $z$ & $z$ \\
$b$ & $b$ & $z$ & $z$ & $z$ & $z$ & $z$ \\
$m$ & $m$ & $z$ & $z$ & $z$ & $z$ & $z$ \\
$z$ & $z$ & $z$ & $z$ & $z$ & $z$ & $z$
\end{tabular}

The reader may verify that $M 1-M 8$ are satisfied. Thus $\mathbb{B}_{1}$ is a residuated lattice in which the ascending chain condition holds. $\mathfrak{B}_{1}$ is obviously nonmodular. Now it is easily verified that D 1 holds in the lattice: $x y \supset\left[x, y^{2}\right]$, 
for every $x, y$ of the lattice. Nevertheless, not every irreducible element is primary. For consider the irreducible $m$. We have $m \supset a b$ and $m \ngtr b$. But since $a$ is idempotent, $m \gg a^{r}$ for any $r$.

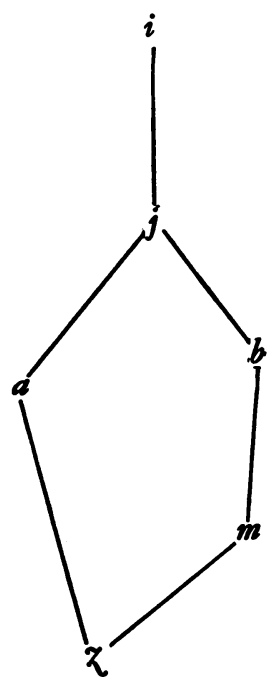

$\mathfrak{B}_{1}$

Next, consider the lattice $\mathfrak{B}_{2}$.

We assign the residuation $x: y$ to $\mathfrak{B}_{2}$ described in Theorem 8.1. The associated multiplication given by Definition 5.1 is then as follows: $x y=y$, if $x=i ; x y=x$ if $y=i ; x y=x$ otherwise.

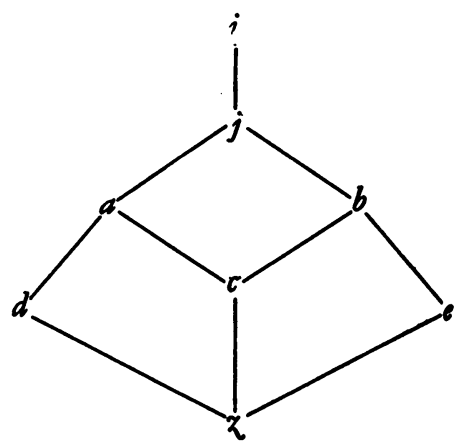

$\mathfrak{B}_{2}$

This lattice is non-modular, as it contains the non-modular sublattice $j, a, d, e, z$. The irreducible elements in it are $j, a, b, d, e$, and these are all primary since $x \supset y^{2}$ for any $y \neq i$ and any $x$. Furthermore, the elements $i, c$, $d, e$, and $z$ are principal and $a=(d, c), b=(c, e), j=(a, b)$. Finally the principal 
elements are closed with respect to multiplication. Thus in this lattice, all hypotheses of Theorem $\mathbf{1 1 . 2}$ hold save modularity; and yet the lattice is a Noether lattice.

Our last example is one in which all the hypotheses of Theorem 11.2 hold save modularity and the lattice is not a Noether lattice. We define a multiplication over $\mathfrak{B}_{3}$ by the following table:

\begin{tabular}{|c|c|c|c|c|c|c|c|c|}
\hline$x\rangle^{y}$ & $i$ & $j$ & $a$ & $b$ & $c$ & $d$ & $m$ & $z$ \\
\hline$i$ & $i$ & $j$ & $a$ & $b$ & $c$ & $d$ & $m$ & $z$ \\
\hline$j$ & $j$ & $d$ & $c$ & $b$ & $m$ & $d$ & $m$ & $z$ \\
\hline$a$ & $a$ & $c$ & $c$ & $z$ & $m$ & $m$ & $m$ & $z$ \\
\hline$b$ & $b$ & $b$ & $z$ & $b$ & $z$ & $b$ & $z$ & $z$ \\
\hline$c$ & $c$ & $m$ & $m$ & $z$ & $m$ & $m$ & $m$ & $z$ \\
\hline$d$ & $d$ & $d$ & $m$ & $b$ & $m$ & $d$ & $m$ & $z$ \\
\hline$m$ & $m$ & $m$ & $m$ & $z$ & $m$ & $m$ & $m$ & $z$ \\
\hline ; & $z$ & $z$ & $z$ & $z$ & $z$ & $z$ & $z$ & $z$ \\
\hline
\end{tabular}

Then it may be verified that the multiplication satisfies $M$ 1-M 8 , and that the elements $i, a, b, c, m, z$ are principal and closed under multiplication.

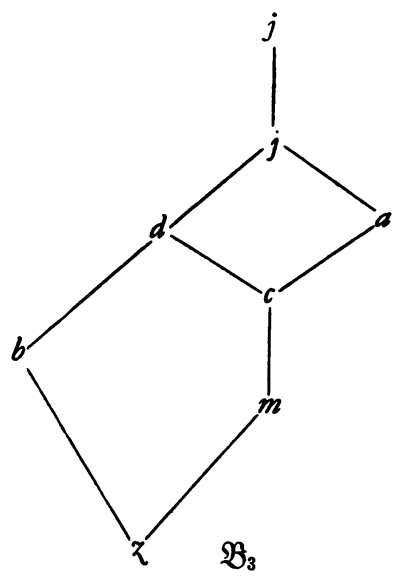

Since $d=(b, m), j=(a, d)$, every element is the union of a finite number of principal elements. The lattice is evidently non-modular. It is not a Noether lattice. For consider the irreducible element $m$. Then $m \supset a b, m \ngtr a$, and $m \ngtr b^{\text {s }}$ for any $s$, since $b$ is idempotent. 


\section{CONDITIONS FOR DISTRIBUTIVITY}

13. We shall conclude by answering some of the questions raised in Ward [4] as to the import of certain auxiliary conditions in a residuated lattice. We consider a residuated lattice in which one or more of the following conditions hold:

R 9. $(a: b, b: a)=i . \quad \mathrm{R} 10 . a:[b, c]=(a: b, a: c) . \quad \mathrm{R} 11 .(b, c): a=(b: a, c: a)$.

THEOREM 13.1. R 9, R 10, R 11 are equivalent and imply distributivity.

$\mathrm{R} 9$ implies $\mathrm{R} 11$. For

$$
\begin{aligned}
(b: a, c: a):\{(b, c): a\} & \supset((b: a):\{(b, c): a\},(c: a):\{(b, c): a\}) \\
& =((b:\{(b, c): a\}): a,(c:\{(b, c): a\}): a) .
\end{aligned}
$$

But $(b:\{(b, c): a\}): a \supset b: c$ since

$$
\begin{aligned}
\{(b:\{(b, c): a\}): a\}:(b: c) & =(\{b:(b: c)\}:\{(b, c): a\}): a \\
& \supset((b, c):\{(b, c): a\}): a \supset a: a=i .
\end{aligned}
$$

Similarly $(c:\{(b, c): a\}): c \supset c: b$. Hence $(b: a, c: a):\{(b, c): a\} \supset(b: c, c: b) \supset i$ by R 9 . Thus $(b: a, c: a) \supset(b, c): a$. But $(b, c): a \supset(b: a, c: a)$ trivially.

$\mathrm{R} 11$ implies R 10 . For by R 11,

$$
\begin{aligned}
(a: b, a: c):\{a:[b, c]\} & =((a: b):\{a:[b, c]\},(a: c):\{a:[b, c]\}) \\
& =((a:\{a:[b, c]\}): b,(a:\{a:[b, c]): c) \\
& \supset([b, c]: b,[b, c]: c) \\
& =(c: b, b: c)=(c:(b, c), b:(b, c))=(c, b):(b, c)=i
\end{aligned}
$$

by $\mathrm{R} 11$. Hence $(a: b, a: c) \supset a:[b, c]$. But $a:[b, c] \supset(a: b, a: c)$ trivially.

$\mathrm{R} 10$ implies R 9. For $(a: b, b: a)=([a, b]: b,[a, b]: a)=[a, b]:[a, b]=i$ by condition $\mathrm{R} 10$.

$\mathrm{R} 10$ implies distributivity. For let $a \supset[b, c]$. Then

$$
a:[(a, b),(a, c)]=(a:(a, b), a:(a, c))=(a: b, a: c)=a:[b, c]=i .
$$

Hence $a \supset[(a, b),(a, c)]$ and $[(a, b),(a, c)] \supset a$ trivially. Therefore $a=[(a, b),(a, c)]$.

THEOREM 13.2. If every element of a residuated lattice is principal, then the lattice is distributive.

Let $(b, c) \supset a$. We have $a \supset([a, b],[a, c])$. Hence

$$
\begin{aligned}
a:(b, c) & \supset([a, b],[a, c]):(b, c)=[([a, b],[a, c]): b,([a, b],[a, c]): c] \\
& \supset[[a, b]: b,[a, c]: c]=[a: b, a: c]=a:(b, c) .
\end{aligned}
$$


Thus $a:(b, c)=([a, b],[a, c]):(b, c)$. But $(b, c) \supset a \supset([a, b],[a, c])$. Hence $a=(a:(b, c))(b, c)=\{([a, b],[a, c]):(b, c)\}(b, c)=([a, b],[a, c])$

by Lemma 13.1 .

Theorem 13.3. A sufficient condition that a residuated lattice with ascending chain condition be a Noether lattice is that every element in it be principal.

For by Theorem 13.2, the lattice is distributive and hence modular; so all the hypotheses of Theorem 11.2 are satisfied.

\section{GARRETT BIRKHOFF}

\section{REFERENCES}

1. On the combination of sub-algebras, Proceedings of the Cambridge Philosophical Society, vol. 39 (1933), pp. 441-464.

2. On the structure of abstract algebras, ibid., vol. 41 (1935), pp. 433-454.

3. Combinatorial relations in projective geometries, Annals of Mathematics, (2), vol. 36 (1935), pp. 743-748.

4. On the lattice theory of ideals, Bulletin of the American Mathematical Society, vol. 40 (1934), pp. 613-619.

R. DedekIND

1. Ueber die von drei Moduln erzeugte Dualgruppe, Gesammelte mathematische Werke, vol. 2, 1931, paper 30, pp. 236-271.

R. P. DILWORTH

1. Abstract residuation over lattices, Bulletin of the American Mathematical Society, vol. 44 (1938), pp. 262-268.

\section{FrITZ KLEIN}

1. Dedekindsche und distributive Verbände, Mathematische Zeitschrift, vol. 41, pp. 261-280.

G. KöTHE

1. Die Theorie der Verbände ..., Jahresbericht der deutschen Mathematiker-Vereinigung, vol. 47 (1937), pp. 125-142.

W. KRULL

1. Axiomatische Begrilndung der allgemeinen Idealtheorie, Sitzungsberichte der physikalischmedicinischen Societät zu Erlangen, vol. 56 (1924), pp. 47-63.

O. ORE

1. On the foundation of abstract algebra, I, Annals of Mathematics, (2), vol. 36 (1935), pp. 406437.

B. L. VAN DER W AERDEN

1. Moderne Algebra, vol. 2, Berlin, 1931.

M. W ARD AND R. P. DILWORTH

1. Residuated lattices, Proceedings of the National Academy of Sciences, vol. 24 (1938), pp. 162164.

M. WARD

1. Residuation in structures over which a multiplication is defined, Duke Mathematical Journal, vol. 3 (1937), pp. 627-636.

2. Structure residuation, Annals of Mathematics, (2), vol. 39 (1938), pp. 558-568.

California Institute of Technology,

Pasadena, Calif. 